\title{
Evaluation of Tools and Techniques for the Generation of Warning Alerts: A Survey Paper
}

\author{
Abid Ghaffar ${ }^{1,3}$, Mohamed Ridza Wahiddin ${ }^{1}$, Mohamad Fauzan Noordin ${ }^{2}$, Asadullah Shaikh ${ }^{4}$ \\ ${ }^{1}$ Department of Computer Science, Kulliyyah of Information and Communication Technology, \\ International Islamic University, Kuala Lumpur, Malaysia. \\ ${ }^{2}$ Department of Computer Science, Kulliyyah of Information and Communication Technology, \\ International Islamic University, Kuala Lumpur, Malaysia. \\ ${ }^{3}$ Department of Computer Science, Foundation Year Program, Umm Al-Qura University, \\ Makkah, Kingdom of Saudi Arabia. \\ ${ }^{4}$ College of Computer Science and Information Systems, \\ Najran University, King Abdulaziz Road, Najran, Saudi Arabia. \\ \{mridza, fauzan\}@iium.edu.my; aaghaffar@uqu.edu.sa; asshaikh@nu.edu.sa
}

\begin{abstract}
Quality assurance is a key factor for the improvement of an organizational behaviour. It is quite challenging to enhance an organizational performance without realising internal errors and mistakes done by its employees. We have also experienced that most of the security solutions are unsuccessful wherever human behaviour is involved. Organisations sometimes pay huge cost for its survival especially when human error is untraceable and misleading. Online survey has been conducted from different professionals serving at different positions in different organisations. Variety of multi-agent system tools (MAS) is available in the market for modelling and simulation of human behaviour. Brahms modelling and simulation tool has been selected among different multi-agent system tools due to its distinguished features to detect human errors in an organisation which supports warning alert generation system.
\end{abstract}

Key Words: Brahms Model, Human behaviour Modelling, Cognitive Science, Security and Privacy, Warning Dialogues, Mental Model Approach

\section{Introduction}

The world is changing and improving day by day with the help of latest developments and research. The real challenge for today's world is to keep in pace of progress and improvement in all the sectors of life. Scientists, technologists, economists, doctors, engineers and professionals are trying their level best to enhance and maintain the quality standards in their existing scenarios. Different sectors of life including agriculture, economy, science, engineering, literature and arts, social sciences need improvement and skills. Every sector of life is involved with human intelligence and behaviour. Human beings are divided into communities and groups based on their religious, social and ethnic beliefs. History proves itself; every impact of society is based on human behavioural changes [17].

Competition in every field of life is a foundation stone for quality assurance and improvement. Every organisation in the existing world wants to improve and produce quality results which are an essential part of its survival. Government and private organisations spend millions of dollars for their own 
improvement and enhancement. Sometimes, huge budget is allocated for the machinery and equipment but results are not encouraging and progressive. The human behaviour plays a key-role for the success and improvement of an organisation [22], [30], [31], [32].

This paper addresses two types of surveys, first survey is conducted among those organisations which have human behavioural problems which results in the form of inefficiency and failure of an organisation, second survey is conducted for the selection of tool based on different parameters to detect human behavioural problems in an organisation.

There are different MAS tools available for modelling and simulation of human behaviour but most of the tools do not cover all the aspects of human behaviour especially when human-machine interaction is concerned. Brahms Model provides holistic approach while dealing with human behavioural roles in an organisation keeping in view of human-machine interaction system [26], [27].

Brahms model provides us an opportunity to address the human behaviour problems in an organisation with the help of monitoring human behaviour activities in an organisation [26], [27]. The model is subdivided into sub-models called agents, objects, artifacts, activity, timing, geography, communication and knowledge. It is used to monitor human behaviour activities and warning codes would be used to generate warning alerts by using warning alert generation system. Once human behavioural errors in an organisation are detected well in time, then corrective measures can be taken to improve the system. Consequently, quality assurance in an organisation can be achieved and maximized by using Brahms model along with warning alert generation system [7], [12], [31], [32].

\subsection{Contributions}

Human behaviour is a key-issue whenever progress of an institution is concerned. Online survey is conducted among different organisations to detect human behaviour problems, which proves that quality assurance problems exist due to human behaviour. Organizational performance is always a keyissue whenever progress of a country is concerned. Online survey proves that different organisations have quality assurance problems due to human behaviour. Organizational performance could be improved and monitored by selecting and using Brahms Modelling and Simulation tool due to its distinguished features which are not available in other MAS [26], [27]. Second survey is conducted for the selection of tool which could detect human behavioural problems in an organisation. Brahms Modelling and Simulation tool is unique due to its completeness in terms of holistic approach. It is found that Brahms Modelling and Simulation tool is ideal to capture human behaviour in any work practice environment. It covers modelling and simulation of human behaviour keeping in view of humanmachine interaction system in an organisation [27]. Comparison between Brahms tools with other multiagent system tools is shown in table 1, which distinguishes the Brahms tool with other MAS tools.

Most of the MAS tools cover one aspect or few aspects of human behaviour in the context of humanmachine interaction. Different parameters like Java support, BDI (Belief, Desire, Intention), goal based, Imperative Programming, Subsumption Languages, Holistic Approach, FIPA (Foundation for Intelligent Physical Agents), Declarative and Reactive features are considered to compare Brahms tool with different MAS like Jason, Agent-Speak, Jade, Jadex, Jack and others. The remainder of the paper is structured as follows: Section II presents a survey questionnaire with results. Section III focuses on the factors involved in choosing a language for agent based modelling and Section IV explores the detail 
Abid Ghaffar, Mohamed Ridza Wahiddin, Mohamad Fauzan Noordin and Asadullah Shaikh; Evaluation of Tools and Techniques for the Generation of Warning Alerts: A Survey Paper. Transactions on Machine Learning and Artificial Intelligence, Volume 3 No 2 April (2015); pp: 10-23

evaluation of existing Brahms modelling and simulations tools. Section V, describes related work. Section VI provides conclusions and identifies directions for future work.

Table 1: $\mathrm{Y}=\mathrm{Yes}, \mathrm{N}=\mathrm{No}$

\begin{tabular}{|l|c|c|c|c|c|c|c|c|c|}
\hline \multicolumn{1}{|c|}{ MAS } & $\begin{array}{c}\text { Java } \\
\text { Support }\end{array}$ & $\begin{array}{c}\text { BDI } \\
\text { Based }\end{array}$ & $\begin{array}{c}\text { Goal } \\
\text { Based }\end{array}$ & Imperative & $\begin{array}{c}\text { Subsumption } \\
\text { Based }\end{array}$ & $\begin{array}{c}\text { Holistic } \\
\text { Approach }\end{array}$ & $\begin{array}{c}\text { FIPA } \\
\text { Standard }\end{array}$ & Declarative & Reactive \\
\hline Brahms & Y & Y & N & N & Y & Y & Y & Y & Y \\
\hline Jason & Y & Y & Y & Y & N & N & Y & Y & Y \\
\hline $\begin{array}{l}\text { Agent } \\
\text { Speak }\end{array}$ & Y & Y & Y & Y & N & N & Y & Y & Y \\
\hline Jade & Y & Y & Y & Y & N & N & Y & Y & Y \\
\hline Jack & Y & Y & Y & Y & N & N & Y & N & Y \\
\hline Jadex & Y & Y & Y & Y & N & N & Y & Y & Y \\
\hline Swarm & Y & N & N & Y & N & N & N & N & Y \\
\hline Repast & Y & N & N & Y & N & N & N & Y & Y \\
\hline NetLogo & Y & N & N & N & N & N & Y & Y & Y \\
\hline 3APL & Y & Y & Y & Y & N & N & Y & Y & Y \\
\hline Prolog & N & N & N & N & N & N & N & Y & Y \\
\hline
\end{tabular}

\section{Survey Report}

A survey has been conducted to identify the problems in an organisation due to human behaviour. The idea is to determine whether the problems exist in different organisations due to human behavioural activities or not. The questions related to human behaviour are included in the survey and designed in such a manner so that human behaviour problems could be detected in an organisation.

A survey is conducted carrying twenty two questions to address the human behaviour issues in different organisations for various positions. More than hundred respondents have participated in the survey through online survey form. An online survey form was distributed among different countries like Saudi Arabia, United Arab Emirates, Qatar, Pakistan, Malaysia, Germany, Norway, United Kingdom and United States of America. Professionals including directors, managers, engineers and many others working at different positions participated in the survey through online submission form system. We have concluded the following points:

- $\quad 75.5 \%$ people work in the organisations for their family and $30 \%$ people work for money.

- $\quad 88 \%$ people want to meet the organizational targets and goals.

- $\quad 85 \%$ people are satisfied from their achieved goals and targets while $9 \%$ people are not satisfied from their job objectives.

- $\quad 43.5 \%$ people use social media up to 2 hours daily.

- $\quad 80 \%$ people try to follow the job rules while $14 \%$ do not follow the job rules.

- $\quad 29 \%$ people suggested that up to $10 \%$ of job rules are not compatible with their job activities while $22 \%$ people suggested that up to $20 \%$ of job rules are not compatible with their job activities.

- $\quad 87 \%$ people responded that they do not have health problems while $7 \%$ people replied that they have health problems. 
- $\quad 85 \%$ people responded that they discuss issues with their colleagues to resolve problems while $8 \%$ people do not like to discuss their problems with their colleagues.

- $\quad 91.5 \%$ responded in the way that colleagues cooperate with them during job activities while $3 \%$ responded negatively in the same sense.

- $\quad 77.7 \%$ replied that they are well trained and skilled based on the job requirements while $17.5 \%$ responded negatively.

- $\quad 77.5 \%$ people suggested they have all the resources while performing job activities while $17.5 \%$ said they do not have enough resources to perform their jobs effectively.

- $\quad 38 \%$ agreed to work for overtime with less salary offer while $57.5 \%$ did not agree to work for overtime without less salary offer.

As a conclusion, the whole survey reveals the larger picture of human behavioural problems which exist in different organisations at different positions and consequently, organizational objectives are compromised. Organisations suffer due to incompatible rules and do not progress due to unskilled and untrained staff. Some people do not interact properly with the colleagues and unable to communicate messages with their colleagues.

\section{Factors involved in choosing a programming language for agent- based modelling}

Agent-based modelling depends upon the specific requirements. General purpose programming languages, specially designed software and toolkits are used to model agents based on the requirements. Agent Based Modelling System (ABMS) can be developed from smaller scale to the larger scale. Desktop computing for ABMS application development includes Spreadsheets using VBA, dedicated agent-based prototyping environments like Repast, NetLogo and StarLogo [19]. General computational mathematical systems include MATLAB and Mathematica [19]. Large scale agent development environments include Repast, Swarm, Mason, AnyLogic and others. General programming languages used for ABMS include Python, Java and C++ [19].

There are different factors involved in the selection of ABMS. We select ABMS based on certain reasons including natural representation of problem, well defined behaviours and decisions, reflection of agent's behaviour from the actual behaviour of individuals, adaption of agents and change of behaviours, agents learning and dynamic interactions and dynamic relationships of agents [19]. It also includes the modelling of process through which agents form organization, having spatial component to their behaviours and interactions and observation of future without involving the past [19].The number of agents, its interactions and states are also considered. Finally, internal structural changes in the model play a vital role for the selection of ABMS [19].

\section{Evaluation of Existing Brahms Modelling and Simulation Tool}

We have considered different agent-based modelling and simulation tools which are available in the market to compare with Brahms modelling and simulation aspects. These tools are AgentSpeak, Jason, Jade, Jack, Jadex, JAM, Swarm, Repast, NetLogo, 3APL, Prolog, Soar and ACT-R. These tools are widely used for modelling and simulation of human behaviour as shown in table 1. Brahms stands for "Business Redesign Agent-based Holistic Modelling System", and now it is being used as modelling language which 
Abid Ghaffar, Mohamed Ridza Wahiddin, Mohamad Fauzan Noordin and Asadullah Shaikh; Evaluation of Tools and Techniques for the Generation of Warning Alerts: A Survey Paper. Transactions on Machine Learning and Artificial Intelligence, Volume 3 No 2 April (2015); pp: 10-23

is comprised of different tools [26], [27]. Brahms provides us the deep insight about human-machine interaction system which helps us to understand how human actually interact with colleagues, documents, and machine, communicate and behave, while performing various job activities [26], [27]. Brahms Process Model comprise of various independent related models which provides us an opportunity to perform modelling job in an easier and efficient way. Brahms uses different models for example, Agents, Objects, Activity, Geography, Timing, Knowledge and Communication which capture all the human-based activities in an organisation [26], [7].

Brahms is considered as an organizational process modelling and simulation tool. Most of multiagent based languages ignore the artifacts and its interaction with environment which makes difficult to develop a holistic model of real-world situations. Brahms actually defines the approach how people and machine work together to accomplish the job by considering behaviours of individuals and groups, how and where communication occurs and synchronization happens in an organisation [26], [27]. Brahms is based on BDI (Belief-Desire-Intention) language and rule-based-system concept [25], [27]. It uses a compiler, a virtual machine to execute Brahms Model, an Eclipse plug-in and an agent viewer program to monitor the activities of different agents at specific interval [23], [27].

According to Brahms modelling and simulation concept, we can record the human behavioural activities in different work practice systems [24]. Brahms is applied in the NASA International Space Stations Mission Control Center (ISSMCC) which is called OCAMS and it is in production since July 2008 [25]. This Program is quite successful despite certain drawbacks which are beyond the control like human mood and emotions.

\subsection{AgentSpeak and Jason}

It is simple but powerful programming language for building rational agents which is based on BDI paradigm. An intellectual heritage of AgentSpeak involves procedural reasoning systems (PRS) which was developed in late 1980's at Stanford Research Institute (SRI) and logic programming (Prolog).

An implementation of AgentSpeak is called Jason which is a development environment for AgentSpeak. Jason is implemented in Java which includes libraries and debugging tools. The main components of AgentSpeak architecture include Beliefs, Intentions, Desires, Plans and Interpreter. Events are initiated towards Interpreter and then Actions are generated by the Interpreter based on the BDI system [6].

Jason is an extension of AgentSpeak language which is goal based and possesses BDI architecture. The main feature includes its support for persistent belief bases, relatively straight forward distribution over a network, high level speech-act based communications layer and plan labels or annotations which can be used to elaborate functions [6], [9].

Referring to the work practice system, Jason is under gone through experimentation phase for the social simulation. It does not cover all the aspects of simulating a complete work practice system specially when agents are interacting with different objects to perform certain activities at certain location and interval [6]. 


\subsection{Jade (Java Agent Development Framework)}

Jade is fully developed in Java and it is used to build agent systems for the management of the networked information resources. It provides a middleware for the development of agent-based applications and can be used in wired and wireless networks. Its main features include interoperability, uniformity, portability, easy to use, and applies the concept of pay-as-you-go-philosophy [8].

Technically, Jade is distributed and multi-party application with peer to peer communication. Jade is object-oriented language and fully compliance with FIPA standards. A single code is written in Jade for database server, application server and presentation client which will work independently and ap $\neg$ plied from end-to-end. Different applications of Jade include collaborative work support, e-learning, eterrorism, network management, knowledge management and entertainment [8].

Simulation of work practice system in an organisation like social human interaction, activities like timing, geography, knowledge, communication and objects altogether is not available in Jade [8].

\subsection{Jack}

It is a commercial and mature product which extends Java in two ways i.e. Syntax and Semantics. Jack defines the top level entities in the form of agent, belief-set, view, event, plan and capability. Applications of Jack include autonomous systems, modelling human-like decision making, decision support applications for military purposes and weather forecasting system [2].

All the features of work practice system which involves human behavioural activities using objects at different situations, locations and timing is not an integral part of Jack [2].

\subsection{Jadex}

It is a combination of XML and Java using the concept of BDI (Belief-Desire-Intention). Jadex agent consist of two components i.e. ADF (Agent Definition File) and procedural plan code. ADF is written in $\mathrm{XML}$ which is based on $\mathrm{BDI}$ and procedural plan code is written in Java. Applications of Jadex include teaching and research, portable PDA-based applications, simulation and scheduling [1].

It does not cover the aspects of human dealing within an organisation using different objects like computers, devices and documents which are useful to perform certain tasks in the work practice system [1].

\subsection{Jam}

It is a Java based language comprised of five primary components i.e. world model which represents database, plan library which represent plans to achieve goals by agents, an interpreter which represents the action plan of agent, an intention structure which represents different activities of agents and an observer which is responsible to access plan in order to achieve desired goals [1], [13].

Jam interpreter selects one plan from a list of applicable plans and places it into the intention structure. An agent may or may not execute the newly selected plan based on the existing intention structure and requirements to execute the plan [13].

Jam does not provide us complete picture of work place practice system. Human-centered activities to complete different tasks using objects and artifacts, is not the part of JAM [13]. 
Abid Ghaffar, Mohamed Ridza Wahiddin, Mohamad Fauzan Noordin and Asadullah Shaikh; Evaluation of Tools and Techniques for the Generation of Warning Alerts: A Survey Paper. Transactions on Machine Learning and Artificial Intelligence, Volume 3 No 2 April (2015); pp: 10-23

\subsection{Swarm}

Swarm intelligence (SI) is an emerging field of biologically-inspired artificial intelligence based on the behavioral models of social insects such as ants, bees, wasps and termites (Bonabeau, 1999).

The main applications include complex interactive virtual environments generation in movie industries, cargo arrangement in airline companies, route scheduling in delivery companies, routing packets in telecommunication networks, power grid optimization control, data clustering, data routing in sensor network, unmanned vehicles controlling in the U.S. military, planetary mapping and micro-satellite controlling in NASA [3], [15], [21].

$\mathrm{SI}$ is based on two basic principles which consist of self-organisation and stigmergy. There are similarities between distributed computing system and social insects. Biologically inspired computing requires identification of analogies, computer modelling of biological mechanisms, and adaptation of biological mechanisms for IT application including motivation and methods [3], [15], [21].

Swarm is also lacking in human centred approach dealing with different objects and artifacts to perform different activities in an organisation [3], [15], [21].

\subsection{Repast}

Repast stands for Recursive Porous Agent Simulation Toolkit (Repast) which is a free open source toolkit mainly developed by Sallach, Collier, Howe, North and others. It is created in the University of Chicago and it was managed by Argonne National Laboratory. Currently Repast is managed by Repast Organization for Architecture and Development (ROAD). Members from government, industrial sector and academic fields participate in the maintenance of Repast [20].

It borrows many ideas from Swarm agent-based modelling toolkit. It has built in adaptive features like genetic algorithms and regression. Repast can be implemented in different computer languages like Python (Repast Py), Java (Repast J), Dot Net (Repast.Net) and C\# which is not possible in Swarm. The main features of Repast include agent templates, fully object-oriented, discrete event scheduling and built-in simulation tools like logging and graphing. It is possible to change the agent behavior equation and agent properties at run time. In addition, Repast provides the support to model the social networking, geographical information system (GIS) and it is available in all modern platforms like Windows, Mac OS and Linux [20].

Modelling and simulation of complete work practice system including agent behaviour and dealing with multiple objects to perform different activities having various parameter is not feasible in Repast [20].

\subsection{NetLogo}

NetLogo is developed by Northwestern University (Center for Connected Learning and Computer-Based Modelling) which is very easy to setup and run models. A very complicated models are outside the capability of NetLogo but successfully used for abstract models. Once models are created, it becomes harder to extend the model. There is encouraging support from academic community and active maintenance is provided by the software developers. Additional features include 3D visualization of models which can be easily embedded in the web pages [28], [29]. 
It is a standalone application written in Java which provides multi-agent programming and modelling environment. It is a member of LISP family and supports agents including library of models with variety of domains [28], [29].

NetLogo world comprised of different goals including four types of agents namely Turtles, Patches, Links and Observer. Turtles are agents that move around two dimensional world which is divided into the form of Patches. Directed or undirected Links are the agents which connect two Turtles. Finally, Observer is an agent which does not possess any location [28], [29].

Complete organisational behaviour keeping human as centre for all the activities using different objects and artifacts is not possible to model and simulate in NetLogo [28], [29].

\subsection{APL}

3APL is an agent-based programming with a variant of modal logic. It is a combination of imperative programming and logic programming [4], [5], [11]. The basic components of 3APL are Goals and Beliefs. Applications of $3 \mathrm{APL}$ include high level control of mobile robots, small device mobile applications and control behaviour of SONY AIBO robots.

3APL does not cover the capability of modelling and simulating human behaviour in an organisation which perform different tasks using different tools like computers, fax-machines, telephones and specific documents [11].

\subsection{Prolog}

It is simply defined as programming logic which is most widely used and inspired by logic. The main features of Prolog are writing facts, querying and writing rules [10], [18]. Prolog can be used as query language for relational database and it can be converted into faster and efficient codes. Declaration of variable names is not required; moreover rules are simple and uniform [18].

On the other hand, prolog is slower and provides an unnatural way to program which can be understood by only expert programmers. It seems clumsy for numerical calculations which cannot be implemented in fast hardware. Prolog cannot be implemented in faster hardware and consumes lot of memory. It also lacks in real time capability.

The main applications of prolog include race track applications, compiler design, digital electronic circuit verification and providing program correctness. Prolog does not provide us complete picture of organisational behaviour in the form of modelling and simulation, where humans are involved in certain activities using different objects and artifacts [10].

\subsection{Soar}

It provides general cognitive architecture for developing systems which possess intelligent behaviour. It carries full range of tasks which can represent and use knowledge properly. Problem solving methods are employed successfully. Different aspects of tasks are learned and its performance is checked and monitored. The combination of different relevant knowledge reveals the decisions at run time process. All decisions made are based on interpretation of sensory data, context of working memory and relevant knowledge. Decisions are not interrupted into the form of sequences [16]. 
Abid Ghaffar, Mohamed Ridza Wahiddin, Mohamad Fauzan Noordin and Asadullah Shaikh; Evaluation of Tools and Techniques for the Generation of Warning Alerts: A Survey Paper. Transactions on Machine Learning and Artificial Intelligence, Volume 3 No 2 April (2015); pp: 10-23

Work practice system in an organisation cannot be modeled and simulated in Soar, where human social behaviour is involved specially performing series of different tasks to achieve targeted goals using different objects is a question mark [16].

\subsection{ACT-R}

It is simply defined as human cognitive architecture, how human thinking-process works actually, and generates knowledge. ACT-R is simulation of understanding human cognition. There is a comparison between ACT-R cognitive modal and fMRI (functional magnetic resonance imaging) dataset which is used to predict the results based on datasets of fMRI [14].

Complete human behavioural modelling and simulation, when human is performing different activities in an organisation using different tools and objects is not available in ACT-R [14].

Most of the tools available in the market for modelling and simulation of human behaviour are limited only to agent-based modelling and simulation as shown in table 1, but Brahms provides us an opportunity not only to model but also simulate complete work practice system in an organisation where human role is significant [24].

\section{Related Work}

Charles M. Macal et al. (2009) presented an idea about agent based modelling system, its application and development process in different aspects of practical scenarios. He focused on ABMS usefulness and its advantage over conventional systems. Applications of ABMS includes Air Traffic Control System, Crime Analysis, Biomedical Research, Chemistry, Epidemic Modelling, Organisational Decision Making and Market Analysis. Different toolkits used for ABMS are Netlogo, Starlogo, Swarm, Repast Simphony, Matlab and Mathematica [19].

Maarten Sierhuis et al. (2002) presented an idea about modelling and simulating work practice system in an organisation using Brahms Model. Brahms Model is comprised of further sub-models called Agents, Objects, Activity, Geography, Timing, Knowledge and Communication. All the activities in a work practice system could be monitored at any interval using Agent Viewer [26].

Chin Seah et al. (2005) gave an idea about the analysis of Brahms model tool by its application in the NASA Mars Exploration Rover (MER) mission. There is a complex situation whenever there is an analysis of work practice system keeping in view of human-machine interaction system. It's quite challenging to model and simulate space mission program using Brahms Model as still there are some areas where we need to improve Brahms modelling and simulating tool [23].

Maarten Sierhuis et al. (2007) presented an idea about detailed version of Brahms Modelling and Simulation technique at the implementation level where more details are needed observe people behaviour and activities in an organisation. It was proved that Brahms Modelling and Simulation technique could be applied in large organisations [27].

Maarten Sierhuis (2013) presented an idea about modelling and simulating multi-agents using Brahms Model which involve people behaviour and their activities at different levels. In contrast, SOAR and ACT$R$ focused on individual agent and its behaviour. Example of one day work practice system was considered to establish the facts about Brahms Modelling and Simulation [25]. 
F. Bellifemine et al. (2003) presented detailed information about the Jade Platform. Main functional-ities of Jade, its architecture and its conceptual model were discussed. Two basic components of its conceptual model comprised of Jade distributed system topology and software component architecture with agent paradigm were explained. Network topology defines, how different components linked together? [8].

Rafael H. Bordini et al. (2006) presented an idea about programming languages and development tools for multi-agent systems. He discussed programming languages issues in terms of declarative, imperative and hybrid nature. He also focused on integrated development environment, platform and framework for these languages [4].

Bijaya Ketan Panigrahi,Yuhui Shi et al. (2011) presented detailed information about biologically inspired algorithms called swarm intelligence. It contains algorithms based on collective individual behaviours and finding its optimal solution. The book covers existing research in its own kind and its real world applications [21].

Mehdi Dastani et al. (2012) presented proceedings of the International Workshop on Programming Multi- Agent Systems (ProMAS 2012) where programming languages and tools for MAS were discussed. Theory and practical issues were discussed in detail in order to analyse different techniques and concepts of multi-agent systems. Revised published papers were presented as source of information [9].

Rafael H. Bordini et al. (2007) presented detailed information about AgentSpeak language using Jason. The book contains explanation about programming multi-agent system using programming language calledAgentSpeak with the help of Jason [6].

Agent Oriented Software Limited Group called AOS group presented white paper carrying detailed information about Jack functionalities and its applications. AOS group focused the application areas where autonomous decision making process is required like Oil Production Systems and Unmanned Vehicles on exploration missions both for under water and space. Jack is autonomous, efficient, resilient and carrying rapid specification [2].

Rafael H. Bordini et al. (2009) presented published papers in the form of book, "Multi-Agent Programming: Languages, Tools and Applications", which comprised of published papers information about multi-agent systems [1].

Alexander Pokahr et al. (2005) presented detailed information about Jadex BDI (Belief, Desire, Intention) reasoning engine. Jadex is a combination of XML (Extensible Markup Language) and Java using BDI concepts. Jadex platform supports editing, debugging and execution of multi-agent systems [1].

Marcus J. Huber et al. (1999) presented idea about JAM architecture and its mobile operational capability due to Java implementation. JAM is a hybrid architecture based on Procedural Reasoning System (PRS), Structured Circuit Semantics (SCS) and Plan-Action combination [13].

Christian Blum et al. (2008) expressed his views about swarm intelligence starting from basic concepts up to the level of real world applications. The book covers foundation concepts of swarm intelligence and presents applications in the field of swarm robotics and telecommunication technology [3].

Aleksandar Jevti' (2011) presented his doctoral thesis about swarm intelligence general framework. He proposed general new design methodology for swarm intelligence tools which did not exist before [15]. 
Abid Ghaffar, Mohamed Ridza Wahiddin, Mohamad Fauzan Noordin and Asadullah Shaikh; Evaluation of Tools and Techniques for the Generation of Warning Alerts: A Survey Paper. Transactions on Machine Learning and Artificial Intelligence, Volume 3 No 2 April (2015); pp: 10-23

Michael J. North et al. (2006) expressed his views about standardization of simulation architecture keeping in view the varying standards followed by different authors. Repast toolkit was examined in different context and suggestions were made for its improvement [20].

Seth Tisue et al. (2004) presented his views about multi-agent language called NetLogo which is being used for education and research. NetLogo is a multi-agent programming language which provides modelling environment for the simulation of complicated phenomenon [28].

Uri Wilensky (1999) presented user manual for NetLogo version 5.0.5 which contains detailed information at the user level. It is in continuous process of development at Center for Connected Learning and Computer-Based Modelling. Instructions can be given to thousands of agents operating at different levels [29].

Fernando Koch et al. (2005) presented his idea about 3APL-M platform which is used to provide multiagent system development environment on mobile devices which has limited resources. BDI and Java language was used in the development of 3APL-M [11].

Ivan Bratko et al. (1986) presented his ideas about programming language which is termed as Prolog. It is non-procedural and declarative language which makes the job easier for the programmer to perform logic operations. The main features include pattern matching, tree-based data structuring and automatic backtracking [18].

Fernando C. N. Pereira et al. (2002) presented his ideas about computational linguistics and logic programming. It focuses on the main concepts of definite clause formalism used in computational linguistics and logic programming [10].

John E. Liard (2012) expressed his views about design and structure of SOAR which is based on complex cognitive architecture. Detailed study has been done, how SOAR works actually and how it respond in a given environment [16].

Jelmer P. Borst et al. (2014) presented his idea about comparison of brain thinking process using ACT-R and FMRI (Functional Magnetic Resonance Imaging). The focus remains in the use of ACT-R architecture using FMRI data [14].

Stephen P. Robbins et al. (2012) expressed his views about the impact of human behaviour in an organisational behaviour. He focused on different aspects of human behaviour in order to enhance the organisational performance [22].

John Preece (2013) presented his idea about human behaviour and its background. He focused on human behavioural roles and its impact on human society [17].

Abid Ghaffar et. al (2013) presented his idea about warning alert generation system using Brahms Mod-elling and Simulation technique. Human behaviour in an organisation could be improved and monitored and rapid action could be taken in the right direction [12].

\section{Conclusion and Future Work}

Organisational performance is compromised due to human behaviour and challenging to address human behavioural roles in terms of performance and improvement. Online survey has been conducted which 
clearly shows human behavioural problems in different organisations. Different MAS tools are evaluated for modelling and simulation of human behaviour in an organisation and we find Brahms modelling and simulation tool is the most appropriate and compatible for the simulation of Work practice system in an organisation. We can generate warning alerts using Brahms Modelling and Simulation tool based on its unique features which is not available in other MAS. MAS have played a significant role in the development of every field of life. Information technology would not be considered consistent, if MAS is not used and applied correctly in the progress of an industry. There is a need to check all the features of MAS in more details as MAS are being developed and updated on regular basis. We can incorporate those changes and developments in our warning alert generation system.

\section{ACKNOWLEDGEMENT}

This research is partially funded by the Malaysian Ministry of Education grant ERGS 11-010-0010 and partially funded by the Umm Al-Qura University, Makkah, Kingdom of Saudi Arabia. We would like to thank Dr. Ghassan Nauman for his useful support.

\section{REFERENCES}

[1] El Fallah Seghrouchni, A., Dix, J., Dastani, M., \& Bordini, R. H. (2009). Multi-Agent Programming. Multi-Agent Programming:: Languages, Tools and Applications, ISBN 978-0-38789298-6. Springer-Verlag US, 2009, 1.

[2] AOS Limited. An Agent Infrastructure for Providing the Decision-Making Capability Required for Autonomous Systems, 2013.

[3] Blum, C., \& Li, X. (2008). Swarm intelligence in optimization (pp. 43-85). Springer Berlin Heidelberg.

[4] Bordini, R. H., Braubach, L., Dastani, M., El Fallah-Seghrouchni, A., Gomez-Sanz, J. J., Leite, J., ... \& Ricci, A. (2006). A survey of programming languages and platforms for multi-agent systems. Informatica (Slovenia), 30(1), 33-44.

[5] Dix, J., \& Seghrouchni, A. E. F. (2005). Multi-Agent Programming. R. H. Bordini, \& M. Dastani (Eds.). Springer Science+ Business Media, Incorporated.

[6] Bordini, R. H., Hübner, J. F., \& Wooldridge, M. (2007). Programming multi-agent systems in AgentSpeak using Jason (Vol. 8). John Wiley \& Sons.

[7] Bravo-Lillo, C., Cranor, L. F., Downs, J. S., \& Komanduri, S. (2011). Bridging the gap in computer security warnings: A mental model approach. IEEE Security \& Privacy, 9(2), 0018-26.

[8] Caire, F. B. G., Poggi, A., \& Rimassa, G. (2003). JADE. A white paper. 
Abid Ghaffar, Mohamed Ridza Wahiddin, Mohamad Fauzan Noordin and Asadullah Shaikh; Evaluation of Tools and Techniques for the Generation of Warning Alerts: A Survey Paper. Transactions on Machine Learning and Artificial Intelligence, Volume 3 No 2 April (2015); pp: 10-23

[9] Dastani, M., Hbner, J. F., \& Logan, B. (2013). Programming Multi-Agent Systems: 10th International Workshop, ProMAS 2012, Valencia, Spain, June 5, 2012, Revised Selected Papers. Springer Publishing Company, Incorporated.

[10] Pereira, F. C., \& Shieber, S. M. (2002). Prolog and natural-language analysis. Microtome Publishing.

[11] Fernando Koch. 3APLM Platform for Deliberative Agents in Mobile Devices, 2005.

[12] Ghaffar, A., Wahiddin, M. R., \& Shaikh, A. (2013). Computer Assisted Alerts Using Mental Model Approach for Customer Service Improvement. Journal of Software Engineering and Applications, 6(05), 21.

[13] Huber, M. J. (1999, April). JAM: A BDI-theoretic mobile agent architecture. In Proceedings of the third annual conference on Autonomous Agents (pp. 236-243). ACM.

[14] Borst, J. P., \& Anderson, J. R. (2014). Using the ACT-R Cognitive Architecture in combination with fMRI data. An Introduction to Model-Based Cognitive Neuroscience. Springer, New York.

[15] Jevtić, A. (2011). Swarm intelligence: novel tools for optimization, feature extraction, and multi-agent system modeling (Doctoral dissertation, Telecomunicacion).

[16] Laird, J. (2012). The Soar cognitive architecture. MIT Press.

[17] J. Preece. A Brief History of Human Behaviour and How to Become an Enlightened Global Citizen (Smashwords Edition, 2013).

[18] Bratko. Prolog Programming For Artificial Intelligence, Addison-Wesley, 1986.

[19] Macal, C. M., \& North, M. J. (2009, December). Agent-based modeling and simulation. In Winter simulation conference (pp. 86-98). Winter Simulation Conference.

[20] North, M. J., Collier, N. T., \& Vos, J. R. (2006). Experiences creating three implementations of the repast agent modeling toolkit. ACM Transactions on Modeling and Computer Simulation (TOMACS), 16(1), 1-25.

[21] Panigrahi, B. K., Shi, Y., \& Lim, M. H. (2011). Handbook of swarm intelligence: concepts, principles and applications (Vol. 8). Springer Science \& Business Media.

[22] Robbins, S., Judge, T. A., Millett, B., \& Boyle, M. (2013). Organisational behaviour. Pearson Higher Education AU.

[23] Seah, C., Sierhuis, M., and J. C., Clancey. Multi-agent modeling and simulation approach for design and analysis of MER Mission Operations. In Proceedings of 2005 International 
conference on Human-Computer interface advances for modeling and simulation (SIMCHI 2005), pages 73-78. Citeseer, 2005.

[24] Sierhuis, M., Modeling and simulating work practice: BRAHMS: A multiagent modeling and simulation language for work system analysis and design. Ph.D Thesis, UvA-DARE, University of Amsterdam (UvA) 2001.

[25] Sierhuis, M. (2013). Multi-agent activity modeling with the Brahms environment. In Theory, Practice, and Applications of Rules on the Web, pages 34-35. Springer Berlin Heidelberg.

[26] Sierhuis, M., \& Clancey, W. J. (2002). Modeling and simulating practices, a work method for work systems design. Intelligent Systems, IEEE, 17(5), 32-41.

[27] Sierhuis, M., Clancey, W. J., \& Van Hoof, R. J. (2007). Brahms: a multi-agent modelling environment for simulating work processes and practices. International Journal of Simulation and Process Modelling, 3(3), 134-152.

[28] Tisue, S., \& Wilensky, U. (2004, May). Netlogo: A simple environment for modeling complexity. In International conference on complex systems (pp. 16-21).

[29] Uri Wilensky. NetLogo User Manual, version 5.0.5, 2013.

[30] M. F. Noordin. (2013). ICT and Islam, IIUM Press.

[31] Ghaffar, A., Wahiddin, M. R., Noordin, M. F., \& Shaikh, A. (2015). A Framework to Improve Customer Service Using Brahms Model. IJEIR, 4(1), 99-106.

[32] Ghaffar, A., Wahiddin, M. R., Shaikh, A., and Ahmad, A. (11-13 Feb. 2015). Generating Alerts using context aware security and Brahms Model for customer service improvement. Accepted paper in International Multi-Topic Conference, Mehran University, Jamshoro, Pakistan. IMTIC'15. 